\title{
Optimal impulse control of a portfolio with a fixed transaction cost
}

\author{
Stefano Baccarin • Daniele Marazzina
}

Published online: 17 May 2013

\begin{abstract}
The aim of this work is to investigate a portfolio optimization problem in presence of fixed transaction costs. We consider an economy with two assets: one risky, modeled by a geometric Brownian motion, and one risk-free which grows at a certain fixed rate. The agent is fully described by his/her utility function and the objective is to maximize the expected utility from the liquidation of wealth at a terminal date. We deal with different forms of utility functions (power, logarithmic and exponential utility), describing in each case how the fixed transaction costs influence the agent's behavior. We show when it is optimal to recalibrate his/her portfolio and which are the best adjusted portfolios. We also analyze how the optimal strategy is influenced by the risk-aversion, as well as other model parameters.
\end{abstract}

Keywords Portfolio optimization · Transaction costs · Impulse control · Quasi-variational inequalities

\section{Introduction}

Portfolio optimization in presence of transaction costs have been intensively studied in literature. For a survey of the literature on consumption-investment problems with transaction costs we refer to Cadenillas (2000). However, most of the articles deal with proportional transaction costs, as, for example, in Davis and Norman (1990),

\section{S. Baccarin}

Dipartimento di Scienze Economico-Sociali e Matematico-Statistiche,

Università degli Studi di Torino, Corso Unione Sovietica 218/bis, 10134 Turin, Italy

e-mail: stefano.baccarin@unito.it

D. Marazzina $(\bowtie)$

Dipartimento di Matematica, Politecnico di Milano, Via Bonardi 9, 20133 Milan, Italy

e-mail: daniele.marazzina@polimi.it 
Shreve and Soner (1994), Akian et al. (1996) and in Liu and Loewenstein (2002), where expected utility maximization problems are considered, or in Assaf et al. (1988), Dumas and Luciano (1991) and in Akian et al. (2001), where the objective is to maximize the long-term growth rate of the portfolio value. In this case, the optimal strategy is to make the minimal effort, in terms of transactions, to maintain the portfolio composition inside a no-trading region. When only one risky stock is present, this means to keep the fraction of the portfolio invested in the risky asset inside an interval of values. The investor trades only when the boundary of the interval is reached, making a great number of small transactions to prevent the portfolio composition to cross the borders (see Davis and Norman 1990; Dumas and Luciano 1991; Shreve and Soner 1994; Liu and Loewenstein 2002). Thus the proposed strategies have a lack of realism because the investor trades continuously, at least part of the time, and he/she makes infinitesimally small transactions, which is not the case in the real world.

Considering the presence of fixed transaction costs leads to more realistic strategies: the agent intervenes only a finite number of times in any time interval and he/ she trades finite amounts of the assets. In Morton and Pliska (1995), Bielecki et al. (2004) authors assume that whenever the investor trades he/she must pay a transaction cost equal to a fixed fraction of the portfolio values ("portfolio management fee'), while Eastham and Hastings (1988), Korn (1998) and Oksendal and Sulem (2002) consider the transaction costs of a trade made of two parts: a fixed fee and a cost proportional to the transaction size. To solve the stochastic control problem, these papers use a dynamic programming approach and the impulse control techniques. Impulse control associate the value function to a Hamilton-JacobiBellman quasi-variational inequal-ity (HJBQVI). In Eastham and Hastings (1988), Korn (1998) authors characterize the value function and the optimal control by means of verification theorems: if a suffi-ciently regular solution of the HJBQVI exists, then that solution is the value function and it is possible to build an optimal control. They solve the problem in some simple examples with a finite horizon, using a linear or, by an asymptotic analysis, an expo-nential utility function. In Oksendal and Sulem (2002) the problem is formulated with an infinite horizon and considering a power utility function with a positive exponent. The value function is characterized as a viscosity solution of the HJBQVI and an example of a numerical solution is given.

In this article we study the optimal strategy for an investor who invests in two assets, one risky and one risk-free. Our agent pays a fixed cost $K>0$ whenever he/ she trades and he/she seeks to maximize the expected value of the utility of his/her terminal wealth at a finite horizon. We formulate the problem as a parabolic impulse control problem where the variables are the values invested in the two assets and time. We will show, in a heuristic approach, that the value function is a solution of the associated HJBQVI and that from this solution it is possible to obtain a Markovian optimal policy. The optimal control is characterized by a continuation and a transaction region, but also by a set of target portfolios. These are the portfolios where it is optimal to move when the portfolio composition touches the borders of the transaction region. Unlike most of the literature on portfolio optimization under transaction costs, see for instance Shreve and Soner (1994), Morton and Pliska (1995), Liu and Loewenstein (2002), Oksendal and Sulem (2002), our optimal policy is not stationary. The control regions change as time passes up to the finite horizon. The main contribution of the present article is to show 
the shape and evolution of the no-transaction region, as well as the target portfolio line, in the presence of a fixed transaction cost and for different utility functions. Moreover we also investigate the sensitivity of the optimal strategy with respect to the model parameters, such as the fixed cost $K$, the risk-aversion of the agent or the volatility of the stock price. We have computed the solution of the HJBQVI numerically by solving (as in Chancelier et al. 2002 and Baccarin 2009) an iterative sequence of variational inequalities, each one representing the value function of a problem where only a finite number of interventions are available. In our numerical experiments we have considered the case of a power or logarithmic utility, which assume a constant relative risk-aversion index for the agent, and the case of an exponential utility, which implies a constant absolute risk-aversion.

The structure of the paper is as follows. In Sect. 2 we describe the financial framework as well as the optimal investment problem in presence of fixed transaction costs. Then, in Sect. 3 we deal with the quasi-variational inequality associated to the opti-mal problem. Section 4 addresses the numerical solution of the problem with a finite element technique, and finally Sect. 5 contains the numerical results.

\section{The investment problem}

We consider a continuous time economy with a finite horizon $T$. We assume that there are two assets: the risk-free asset $B$ with a constant instantaneous interest rate $r$, and a risky asset $S$, whose price evolves according to a geometric Brownian motion of constant drift $b$ and volatility $\sigma$

$$
d S(t)=b S(t) d t+\sigma S(t) d Z(t)
$$

$Z_{t}$ being an adapted Wiener process on the filtered probability space $\left(\Omega, F, P, \mathbb{F}_{t}\right)$. The agent maximizes the expected utility over the finite horizon. The utility at time $T$ is a function of the wealth, and we assume one of the following forms for the utility function:

$$
\begin{aligned}
& u(x)=\frac{x^{\gamma}}{\gamma}, \quad \gamma<0 \text { or } 0<\gamma<1, \\
& u(x)=\log (x), \\
& u(x)=-e^{-\gamma x}, \quad \gamma>0 .
\end{aligned}
$$

We will denote the investor's portfolio by

$$
\left(\pi_{S}, \pi_{B}\right)
$$

where $\pi_{S}$ and $\pi_{B}$ are the amounts of money invested respectively in the stock and in the risk-free asset. At any time $t$ the agent can buy (or sell if $\xi<0$ ) a value $\xi$ of the risky asset reducing (or increasing) correspondingly the bank account. However, for each transaction, he/she must face a fixed transaction cost, paying $K$. Thus the portfolio composition in $t$ becomes 


$$
\left(\pi_{S}\left(t^{-}\right)+\xi, \pi_{B}\left(t^{-}\right)-K-\xi\right)
$$

where $\left(\pi_{S}\left(t^{-}\right), \pi_{B}\left(t^{-}\right)\right)$are the values invested in the two assets just before the transaction.

A fundamental notion in our model is the liquidation value of the portfolio. We define the liquidation value $L\left(\pi_{S}, \pi_{B}\right)$ as

$$
L\left(\pi_{S}, \pi_{B}\right)=\pi_{S}+\pi_{B}-K \quad \text { if } \pi_{S} \neq 0, \quad L\left(0, \pi_{B}\right)=\pi_{B}
$$

i.e., it is the value when the long or short position in the risky asset is cleared out. Besides the transaction costs, we assume that the agent must face a solvency constraint, that is a portfolio is admissible only if $L\left(\pi_{S}(t), \pi_{B}(t)\right) \geq L_{\min }, \forall t \in[0, T]$. Here $L_{\min }$ is a positive constant denoting the minimum value of liquidity required to the agent. If his/her wealth reaches this lower bound the portfolio will be liquidated and the remaining value invested only in the risk-free asset. Moreover we will assume that there are lower bounds $B_{\min }<0$ and $S_{\min }<0$ in the open short positions in the bank account and in the risky security, respectively. We thus define the region $\mathcal{P} \subset \mathbb{R}^{2}$ of admissible portfolios by

$$
\mathcal{P}=\left\{\left(\pi_{S}, \pi_{B}\right) \in \mathbb{R}^{2}: L\left(\pi_{S}, \pi_{B}\right) \geq L_{\min } \wedge\left(B \geq B_{\min }\right) \wedge\left(S \geq S_{\min }\right)\right\} .
$$

A control policy $p$ is a sequence of stopping times $\left\{\tau_{i}\right\}$ (with respect to the filtration $\mathbb{F}_{t}$ ) and corresponding random variables $\left\{\xi_{i}\right\}$ verifying the conditions:

$$
\left\{\begin{array}{l}
0 \leq \tau_{i} \leq \tau_{i+1} \text { almost surely } \forall i \\
\lim _{i \rightarrow+\infty} \tau_{i}=+\infty \text { almost surely } \\
\xi_{i} \text { is } F_{\tau_{i}} \text { measurable }
\end{array}\right.
$$

Here $\xi_{i}$ represents the value of stocks bought (if $\xi_{i}>0$ ) or sold (if $\xi_{i}<0$ ) at time $\tau_{i}$. Notice that $\lim _{i \rightarrow \infty} \tau_{i}=\infty$ implies that the number of stopping times $\tau_{i}$ which are less or equal to $T$ is almost surely finite $\left(\tau_{i}=+\infty\right.$ almost surely for some $i<\infty$ is possible). Starting from the initial condition $\left(\pi_{B}(0), \pi_{S}(0)\right)$ the dynamics of the controlled portfolio can be described by the following set of stochastic differential equations:

$$
\begin{aligned}
& \left\{\begin{array}{l}
d \pi_{S}=b \pi_{S} d t+\sigma \pi_{S} d Z \\
d \pi_{B}=r \pi_{B} d t
\end{array} \quad \text { if } \tau_{i}<t<\tau_{i+1}\right. \\
& \left\{\begin{array}{l}
\pi_{S}\left(\tau_{i}\right)=\pi_{S}\left(\tau_{i}^{-}\right)+\xi_{i} \\
\pi_{B}\left(\tau_{i}\right)=\pi_{B}\left(\tau_{i}^{-}\right)-\xi_{i}-K
\end{array} \quad \text { if } t=\tau_{i},\right.
\end{aligned}
$$

$Z$ being the one-dimensional Brownian motion driving the risky asset.

We will say that a policy $p$ is admissible if the corresponding controlled process verifies $\left(\pi_{B}^{p}(t), \pi_{S}^{p}(t)\right) \in \mathcal{P}, \forall t \in[0, T]$. Since the investor's preferences are represented by one of the utility functions (1), we can formulate our model as the following optimal impulse control problem 


$$
\max _{p \in A\left(0, \pi_{S}(0), \pi_{B}(0)\right)} E_{0, \pi_{S}(0), \pi_{B}(0)}\left[u\left(L\left(\pi_{S}(T), \pi_{B}(T)\right)\right]\right.
$$

where $A\left(0, \pi_{S}(0), \pi_{B}(0)\right)$ is the set of admissible policies when the process starts at time 0 with a portfolio $\left(\pi_{S}(0), \pi_{B}(0)\right)$.

We will solve this problem by using a dynamic programming approach, considering a variable initial condition and the value function

$$
V\left(t, \pi_{S}, \pi_{B}\right)=\sup _{p \in A\left(t, \pi_{S}, \pi_{B}\right)} E_{t, \pi_{S}, \pi_{B}}\left[u\left(L\left(\pi_{S}(T), \pi_{B}(T)\right)\right]\right.
$$

defined in $[0, T] \times \mathcal{P}$. Here $A\left(t, \pi_{S}, \pi_{B}\right)$ is the set of admissible policies when the process starts in $t$ with a portfolio $\left(\pi_{S}, \pi_{B}\right)$. In the next section we will show heuristically that $V\left(t, \pi_{S}, \pi_{B}\right)$ is a solution of a quasi-variational inequality, and that there exists an optimal control of a Markovian type for our model.

\section{The quasi-variational inequality associated to the value function and the optimal control}

We define the following non-local operator $\mathcal{M}$ for bounded functions in $[0, T] \times \mathcal{P}$ as

$$
\mathcal{M} V:=\sup _{\xi \in F\left(\pi_{S}, \pi_{B}\right)} V\left(t, \pi_{S}+\xi, \pi_{B}-\xi-K\right)
$$

being $F\left(\pi_{S}, \pi_{B}\right)$ the set of admissible transactions from $\left(\pi_{S}, \pi_{B}\right) \in \mathcal{P}$

$$
F\left(\pi_{S}, \pi_{B}\right):=\left\{\xi \in \mathbb{R}:\left(\pi_{S}+\xi, \pi_{B}-\xi-K\right) \in \mathcal{P}\right\} .
$$

Notice that $\mathcal{M V}$ corresponds to the best transaction the agent can make if he/she decides to intervene. If $F\left(\pi_{S}, \pi_{B}\right)=\emptyset$, we set $\mathcal{M} V=-\infty$. We also define the second order linear operator $\mathcal{L}$ by

$$
\mathcal{L} V:=\frac{\partial V}{\partial t}+r \pi_{B} \frac{\partial V}{\partial \pi_{B}}+b \pi_{S} \frac{\partial V}{\partial \pi_{S}}+\frac{1}{2} \sigma^{2} \pi_{S}^{2} \frac{\partial^{2} V}{\partial \pi_{S}^{2}} .
$$

In this section we will show, in a formal way, that the value function $V$ of our problem is a solution of the following parabolic quasi-variational inequality in $(0, T) \times \mathcal{P}$

$$
\begin{aligned}
& V\left(t, \pi_{S}, \pi_{B}\right) \geq \mathcal{M} V\left(t, \pi_{S}, \pi_{B}\right) \\
& \mathcal{L} V\left(t, \pi_{S}, \pi_{B}\right) \leq 0 \\
& \left(V\left(t, \pi_{S}, \pi_{B}\right)-\mathcal{M} V\left(t, \pi_{S}, \pi_{B}\right)\right) \mathcal{L} V\left(t, \pi_{S}, \pi_{B}\right)=0 .
\end{aligned}
$$

We will assume that the value function is regular enough to apply the Dynkin's formula in $(0, T)$. This is the case when the value function is $C^{1,2}$ but it can also hold true when its distributional derivatives are ordinary functions in Sobolev spaces. Let 
us consider our agent at the time instant $t$. He/she can take only one of two possible decisions:

1) to let the system evolve freely for the infinitesimal interval $(t, t+h)$;

2) to make the best transaction, selling or buying stocks.

Since there is no other alternative it is likely that the following version of Bellman's optimality principle holds true:

$V\left(t, \pi_{S}, \pi_{B}\right)=\max \left\{E_{t, \pi_{S}, \pi_{B}}\left[V\left(t+h, \pi_{S}(t+h), \pi_{B}(t+h)\right)\right], \mathcal{M} V\left(t, \pi_{S}, \pi_{B}\right)\right\}$.

Therefore we obtain immediately $V \geq \mathcal{M} V$, which is condition (2). Now, suppose that we can apply the Dynkin's formula in the interval $(t, t+h)$. We obtain

$$
\begin{aligned}
& E_{t, \pi_{S}, \pi_{B}}\left[V\left(t+h, \pi_{S}(t+h), \pi_{B}(t+h)\right)\right] \\
& \quad=V\left(t, \pi_{S}, \pi_{B}\right)+E_{t, \pi_{S}, \pi_{B}}\left[\int_{t}^{t+h} \frac{\partial V}{\partial s}+r \pi_{B} \frac{\partial V}{\partial \pi_{B}}+b \pi_{S} \frac{\partial V}{\partial \pi_{S}}+\frac{1}{2} \sigma^{2} \pi_{S}^{2} \frac{\partial V}{\partial \pi_{S}^{2}} d s\right] .
\end{aligned}
$$

But, from the Bellman's principle, it holds

$$
V\left(t, \pi_{S}, \pi_{B}\right) \geq E_{t, \pi_{S}, \pi_{B}}\left[V\left(t+h, \pi_{S}(t+h), \pi_{B}(t+h)\right)\right]
$$

and consequently we have

$$
E_{t, \pi_{S}, \pi_{B}}\left[\int_{t}^{t+h} \frac{\partial V}{\partial s}+r \pi_{B} \frac{\partial V}{\partial \pi_{B}}+b \pi_{S} \frac{\partial V}{\partial \pi_{S}}+\frac{1}{2} \sigma^{2} \pi_{S}^{2} \frac{\partial V}{\partial \pi_{S}^{2}} d s\right] \leq 0 .
$$

Letting $h \rightarrow 0^{+}$and using the integral version of the mean value theorem we obtain the inequality (3), that is $\mathcal{L} V \leq 0$. Since no other alternative is possible, the third equality (4), $(V-\mathcal{M} V) \mathcal{L} V=0$, is also verified. To uniquely characterize $V$ as a solution of $(2-4)$ in $[0, T] \times \mathcal{P}$ we must consider the behavior of the value function at the boundary of $(0, T) \times \mathcal{P}$. At the terminal date $T$ it holds, obviously, $V$ $\left(T, \pi_{S}, \pi_{B}\right)=u\left(L\left(\pi_{S}, \pi_{B}\right)\right), \forall\left(\pi_{S}, \pi_{B}\right) \in \mathcal{P}$. Along the straight line $\pi_{S}+\pi_{B}=K+$ $L_{\text {min }}$, we have

$$
V\left(t,-\pi_{B}+K+L_{\min }, \pi_{B}\right)=u\left(L_{\min } e^{r(T-t)}\right) \forall t \in[0, T]
$$

because the minimum solvency level has been reached and the investor is forced to liquidate his/her position. Moreover when $\pi_{B}=B_{\min }$ or $\pi_{S}=S_{\min }$ we have $V=\mathcal{M V}$ because one of the open short position is too big and the agent is forced to transact. The value function is also determined by the fact that it is upper bounded, because it is certainly lower than the value function of a corresponding problem without transaction costs. 
We also can show heuristically that an optimal control of a Markovian type always exists for our model. We divide the $[0, T] \times \mathcal{P}$ domain into two regions, the transaction region

$$
A \equiv\left\{\left(t, \pi_{S}, \pi_{B}\right) \in[0, T] \times \mathcal{P}: V=\mathcal{M} V\right\}
$$

and the complementary continuation region

$$
C \equiv\left\{\left(t, \pi_{S}, \pi_{B}\right) \in[0, T] \times \mathcal{P}: V>\mathcal{M} V\right\}
$$

Setting $\tau_{0}^{*} \equiv t$, the optimal policy $p^{*}\left(t, \pi_{S}, \pi_{B}\right)$ for the process starting in $\left(t, \pi_{S}, \pi_{B}\right)$ is given by:

$$
p^{*}=\left\{\begin{array}{l}
\tau_{i}^{*}=\left\{\begin{array}{l}
\inf \left\{I_{i} \equiv\left\{T \geq s \geq \tau_{i-1}^{*}:\left(s, \pi_{S}\left(s^{-}\right), \pi_{B}\left(s^{-}\right)\right) \in A\right\}\right\} \\
+\infty \text { if } I_{i}=\emptyset
\end{array}\right. \\
\xi_{i}^{*}=\left\{\begin{array}{l}
\arg \max \quad V\left(\tau_{i}, \pi_{S}\left(\tau_{i}^{*-}\right)+\xi, \pi_{B}\left(\tau_{i}^{*-}\right)-\xi-K\right) \\
\xi \in F\left(\pi_{S}\left(\tau_{i}^{-}\right), \pi_{B}\left(\tau_{i}^{-}\right)\right) \\
\operatorname{arbitrary} \text { if } \tau_{i}^{*}=+\infty
\end{array}\right.
\end{array}\right.
$$

Indeed, if we apply the Dynkin's formula, separately in the intervals $\left(\tau_{i-1}^{*} \wedge T, \tau_{i}^{*} \wedge T\right)$ to the process $\left(\pi_{S}^{*}, \pi_{B}^{*}\right)$ controlled by policy $p^{*}$, and we take account of the jumps $\xi_{i}^{*}$, we have $(i=1, \ldots, m)$ :

$$
\begin{aligned}
& E_{t, \pi_{S}, \pi_{B}}\left[V\left(\tau_{m}^{*} \wedge T, \pi_{S}\left(\tau_{m}^{*-} \wedge T\right), \pi_{B}\left(\tau_{m}^{*-} \wedge T\right)\right)\right]=V\left(t, \pi_{S}, \pi_{B}\right) \\
& +E_{t, \pi_{S}, \pi_{B}}\left[\sum_{i=0}^{m-1} \int_{\tau_{i}^{*} \wedge T}^{\tau_{i+1}^{*} \wedge T}\left(\frac{\partial V}{\partial s}+r \pi_{B} \frac{\partial V}{\partial \pi_{B}}+b \pi_{S} \frac{\partial V}{\partial \pi_{S}}+\frac{1}{2} \sigma^{2} \pi_{S}^{2} \frac{\partial V}{\partial \pi_{S}^{2}}\right) d s\right] \\
& +E_{t, \pi_{S}, \pi_{B}}\left[\sum _ { i = 1 } ^ { m - 1 } \left(V\left(\tau_{i}^{*}, \pi_{S}\left(\tau_{i}^{*-}\right)+\xi_{i}^{*}, \pi_{B}\left(\tau_{i}^{*-}\right)-\xi_{i}^{*}-K\right)\right.\right. \\
& \left.-V\left(\tau_{i}, \pi_{S}\left(\tau_{i}^{*-}\right), \pi_{B}\left(\tau_{i}^{*-}\right)\right) \chi_{\tau_{i}^{*}<\infty}\right] .
\end{aligned}
$$

Since $V$ verifies $\mathcal{L} V=0$ when $V>\mathcal{M} V$, and by construction $\left(s, \pi_{S}^{*}, \pi_{B}^{*}\right) \in C$ in the intervals $\left(\tau_{i}^{*} \wedge T, \tau_{i+1}^{*} \wedge T\right)$ when $\tau_{i}^{*} \wedge T<\tau_{i+1}^{*} \wedge T$, all the terms in the first expectation vanish. Similarly, as $V$ verifies $V=\mathcal{M} V$ in $A$, and by construction $\left(\tau_{i}^{*}, \pi_{S}\left(\tau_{i}^{*-}\right), \pi_{B}\left(\tau_{i}^{*-}\right)\right) \in A$ if $\tau_{i}^{*}<\infty$, we have $V\left(\tau_{i}^{*}, \pi_{S}\left(\tau_{i}^{*-}\right)+\xi_{i}^{*}, \pi_{B}\left(\tau_{i}^{*-}\right) . \xi_{i}^{*}-\right.$ $K)_{\tau_{i}^{*}<\infty}=V\left(\tau_{i}^{*}, \pi_{S}\left(\tau_{i}^{*-}\right), \pi_{B}\left(\tau_{i}^{*-}\right)_{\tau_{i}^{*}<\infty}\right.$, and also the second expectation vanishes. Therefore we obtain

$$
V\left(t, \pi_{S}, \pi_{B}\right)=E_{t, \pi_{S}, \pi_{B}}\left[V\left(\tau_{m}^{*} \wedge T, \pi_{S}\left(\tau_{m}^{*-} \wedge T\right), \pi_{B}\left(\tau_{m}^{*-} \wedge T\right)\right)\right]
$$


By taking the limit for $m \rightarrow \infty$, as $\tau_{m}^{*} \rightarrow \infty$ almost surely because $p^{*}$ is admissible, we have

$$
V\left(t, \pi_{S}, \pi_{B}\right)=E_{t, \pi_{S}, \pi_{B}}\left[V\left(T, \pi_{S}^{*}(T), \pi_{B}^{*}(T)\right)\right]=E_{t, \pi_{S}, \pi_{B}}\left[U\left(L\left(\pi_{S}^{*}(T), \pi_{B}^{*}(T)\right)\right)\right]
$$

and by the definition of the value function the policy $p^{*}$ is optimal.

\section{A numerical solution}

To solve numerically our model, it is necessary to deal with a finite domain. To avoid artificial boundary conditions, instead of using some transformation to reduce the unbounded region $\mathcal{P}$ to a bounded domain, we will assume that the agent must face another constraint, which has a natural economic meaning. Our investor will be fully satisfied if his/her portfolio reaches a threshold liquidation value $L_{\max }$, at a time $t<T$. In this case the portfolio will be liquidated in $t$ and $L_{\max }$ will be invested in the bank account up to the finite horizon $T$. Therefore we can define the bounded region $\mathcal{P}_{h} \subset \mathcal{P}$ of admissible portfolios by

$$
\mathcal{P}_{h}=\left\{\left(\pi_{S}, \pi_{B}\right) \in \mathbb{R}^{2}:\left(L_{\min } \leq L\left(\pi_{S}, \pi_{B}\right) \leq L_{\max }\right) \wedge\left(\pi_{B} \geq B_{\min }\right) \wedge\left(\pi_{S} \geq S_{\min }\right)\right\}
$$

The bounded region $\mathcal{P}_{h}$ is depicted in Fig. 1: $\mathcal{P}_{h}$ consists of the trapezoidal domain $\mathrm{ABCD}$ and the segment GF. For computational purposes, we only consider the bounded domain ABCD.

We now consider the backward-in-time problem: as specified in the previous section, at the final time $T$ it holds

$$
V\left(T, \pi_{S}, \pi_{B}\right)=u\left(L\left(\pi_{S}, \pi_{B}\right)\right) .
$$

The value function is fully described by the terminal condition problem (2-4). We set the boundary conditions on the domain depicted in Fig. 1 as follows:

- on edges $\mathrm{AB}$ and $\mathrm{CD}: V\left(t, \pi_{S}, \pi_{B}\right)=u\left(L\left(\pi_{S}, \pi_{B}\right) e^{r(T-t)}\right)$;

- on edges BC and AD: $V\left(t, \pi_{S}, \pi_{B}\right)=\mathcal{M} V\left(t, \pi_{S}, \pi_{B}\right)$.

The first boundary condition is due to the assumption on the threshold liquidation values $L_{\min }$ and $L_{\max }$ : if the agent reaches these levels, he/she recalibrate his/her portfolio investing only in the risk-free asset up to the horizon $T$. The second boundary condition is due to the $B_{\min }$ and $S_{\min }$ bounds, where the agent is obliged to transact.

The above problem can be solved with a projected SOR method coupled with an iterative procedure above the obstacle (2-4). We define the function $V_{0}\left(t, \pi_{S}, \pi_{B}\right)$ such that

$$
\mathcal{L} V_{0}\left(t, \pi_{S}, \pi_{B}\right)=0
$$


Fig. 1 Bounded admissible region $\left(\mathcal{P}_{h}\right)$ : OG $=K$, $\mathrm{OF}=\mathrm{OE}=K+L_{\min }$, while the trapezoidal region has vertices $\mathrm{A}=\left(B_{\min }, K+\right.$ $\left.L_{\min }-B_{\min }\right), \mathrm{B}=\left(K+L_{\min }-\right.$ $\left.S_{\min }, S_{\min }\right), \mathrm{C}=\left(K+L_{\max }-\right.$ $\left.S_{\min }, S_{\min }\right)$ and $\mathrm{D}=\left(B_{\min }\right.$, $\left.K+L_{\max }-B_{\min }\right)$

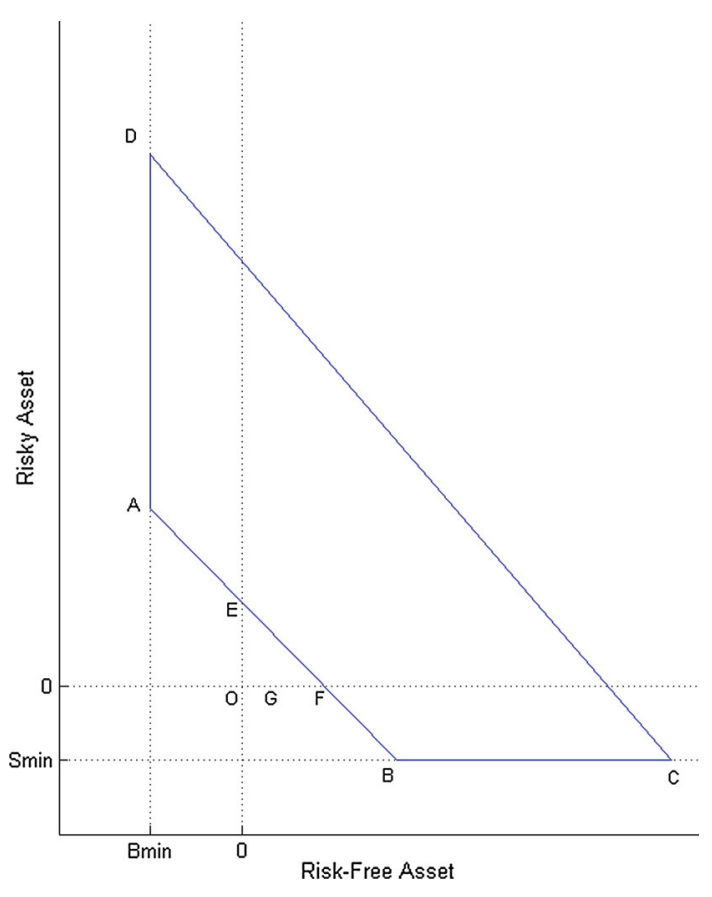

for any $t \in[0, T)$ and $\left(\pi_{S}, \pi_{B}\right) \in \mathcal{P}_{h}$, with the condition $V\left(t, \pi_{S}, \pi_{B}\right)=$ $u\left(L\left(\pi_{S}, \pi_{B}\right) e^{r(T-t)}\right)$ on the whole boundary. This is the the expected utility of the final position when no transactions are permitted. Beginning with $V_{0}\left(t, \pi_{S}, \pi_{B}\right)$, one defines $V_{i}\left(t, \pi_{S}, \pi_{B}\right), i \geq 1$, as the solution of

$$
\begin{aligned}
& V_{i}\left(t, \pi_{S}, \pi_{B}\right) \geq \mathcal{M} V_{i-1}\left(t, \pi_{S}, \pi_{B}\right), \\
& \mathcal{L} V_{i}\left(t, \pi_{S}, \pi_{B}\right) \leq 0, \\
& \left(V_{i}\left(t, \pi_{S}, \pi_{B}\right)-\mathcal{M} V_{i-1}\left(t, \pi_{S}, \pi_{B}\right)\right) \mathcal{L} V_{i}\left(t, \pi_{S}, \pi_{B}\right)=0,
\end{aligned}
$$

with boundary condition $V_{i}\left(t, \pi_{S}, \pi_{B}\right)=\mathcal{M} V_{i-1}\left(t, \pi_{S}, \pi_{B}\right)$ on edges BC and AD. In the Appendix we show, in a formal way, that the solution $V_{i}\left(t, \pi_{S}, \pi_{B}\right)$ can be interpreted as the value function of our problem when at most $i$ transaction can be made by the investor. Thus it holds

$$
V_{0}\left(t, \pi_{S}, \pi_{B}\right) \leq V_{1}\left(t, \pi_{S}, \pi_{B}\right) \leq V_{2}\left(t, \pi_{S}, \pi_{B}\right) \leq \cdots \leq V\left(t, \pi_{S}, \pi_{B}\right),
$$

and, as the number of transactions in the interval $[0, T]$ is almost surely finite, the sequence $V_{i}$ converges to $V$.

Each variational inequality (7) can be solved with a projected SOR method. We discretize the PDE $\mathcal{L} V=0$ considering a finite element technique with polynomial of degree 1, and a Crank-Nicholson scheme. For details on the implementation of the PSOR algorithm see, for example, Wilmott et al. (1995), while for applications of the finite element technique to financial problems see, as examples, Achdou and Pironneau (2005), Barucci and Marazzina (2012), Marazzina et al. (2012). 


\section{Numerical results}

In this section we present some numerical results considering different utility functions and different values of the model parameters. We will always assume a finite horizon of one year $(T=1)$ and the following bounds of the domain (see Fig. 1): $B_{\min }=S_{\min }=$ $-20, L_{\min }=1$ and $L_{\max }=100$. The finite element discretization is done with a mesh of 3,000 points (approximately 5,000 triangles) and a time grid of 50 steps. The numerical experiments are performed with Matlab R2011a. We will separate the case of a power or logarithmic utility, with a constant relative risk-aversion, from the case of an exponential utility, with a constant absolute risk-aversion. In both cases an exact solution is available if we consider our portfolio problem without transaction costs.

\subsection{Power and logarithmic utility}

In this case it is well known that the optimal policy without transaction costs is to transact continuously, to maintain fixed the proportion of wealth invested in the risky asset

$$
\frac{\pi_{S}}{\pi_{B}+\pi_{S}}=\frac{b-r}{\sigma^{2}(1-\gamma)}
$$

the so-called Merton's proportion, see Merton (1969). The logarithmic utility can be seen as the particular case when $\gamma=0$. To analyze the influence of transaction costs, in Figs. 2, 3, 4 and 5 we show the transaction region (in blue) as well as the target portfolios (in red), i.e., the portfolio where it is optimal to move when the agent portfolio falls into the transaction region. In these figures we have set the values $r=$ $3 \%, b=0.08, \sigma=0.5$, we have considered a power utility with exponent $\gamma=0.5$ and different transaction costs $(K=0.01,0.05,0.1$ and 0.25$)$. We show the transaction areas at time $t=0$ and $t=0.5$. From these numerical experiments we notice that the target portfolios belongs to the Merton's line, with few exceptions near the edge CD (see Fig. 1). These exceptions are due to the fact that the portfolio value is already near the threshold value $L_{\max }$. The investor will likely liquidate his/her position in short time and before $T$, and this makes the risky asset less attractive. The transaction region consists of two parts. The shape of the continuation region (white) is similar to a cone, enlarging as time increases, and it seems nearly symmetric with respect to the Merton's line.

Moreover, as expected, transaction costs strongly influence the optimal strategies. The transaction region, in fact, decreases as the transaction cost $K$ increases. Moreover, it also decreases as time increase: this happens because, as the time to maturity $T-t$ decreases, only a large change in the portfolio composition can compensate the transaction costs.

To describe how the risk-aversion influences the transaction region as well as the target portfolio, in Fig. 6 we consider both the power utility function (with different exponents) as well as the logarithmic utility. Considering this figure and Fig. 4 (left), we notice that, as expected, increasing the risk-aversion $1-\gamma$, the target portfolios move according to the Merton's line, i.e., the agent prefers to invest more in the risk-free 

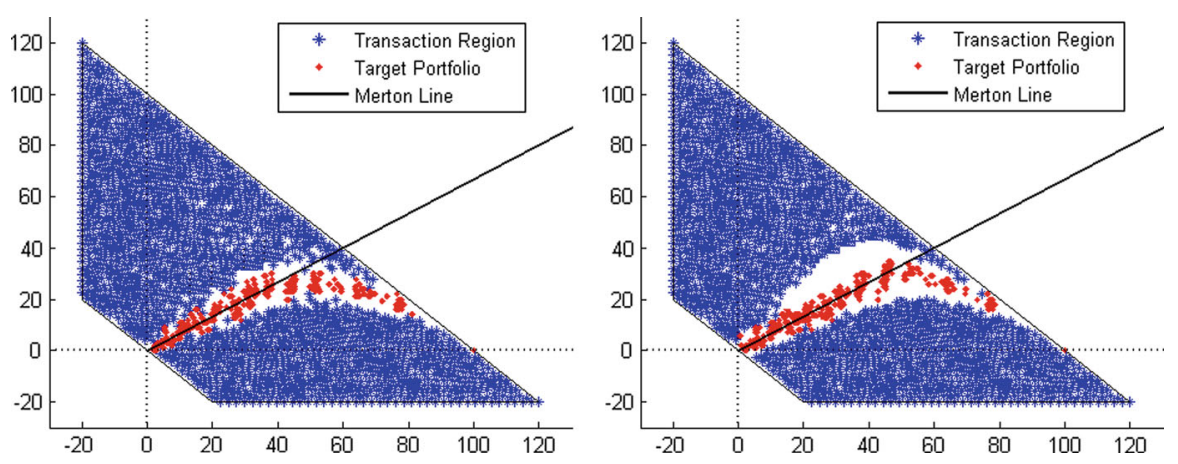

Fig. 2 Transaction area in the plane $\left(\pi_{B}, \pi_{S}\right)$ for power utility. Time $t=0$ (left) and $t=0.5$ (right). $K=0.01$
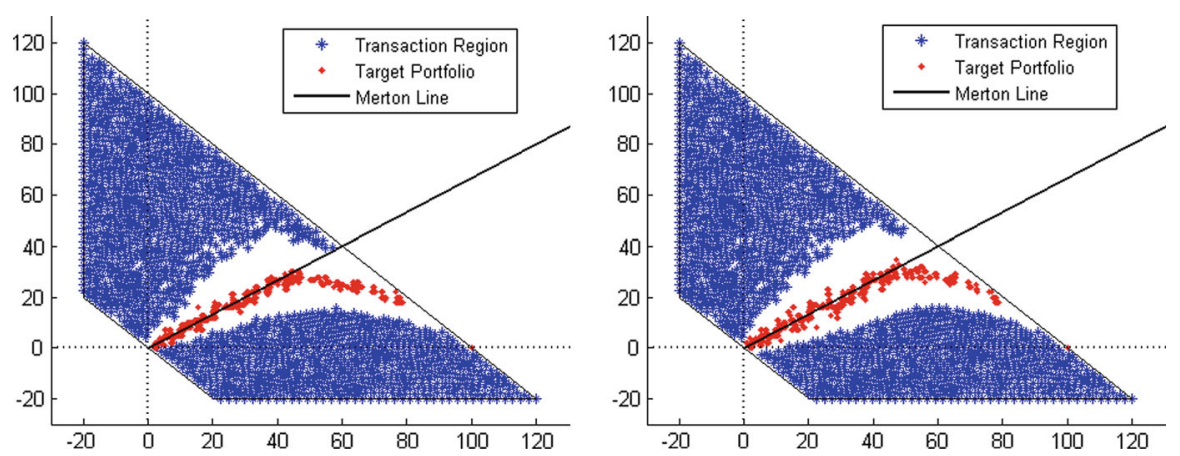

Fig. 3 Transaction area in the plane $\left(\pi_{B}, \pi_{S}\right)$ for power utility. Time $t=0$ (left) and $t=0.5$ (right). $K=0.05$
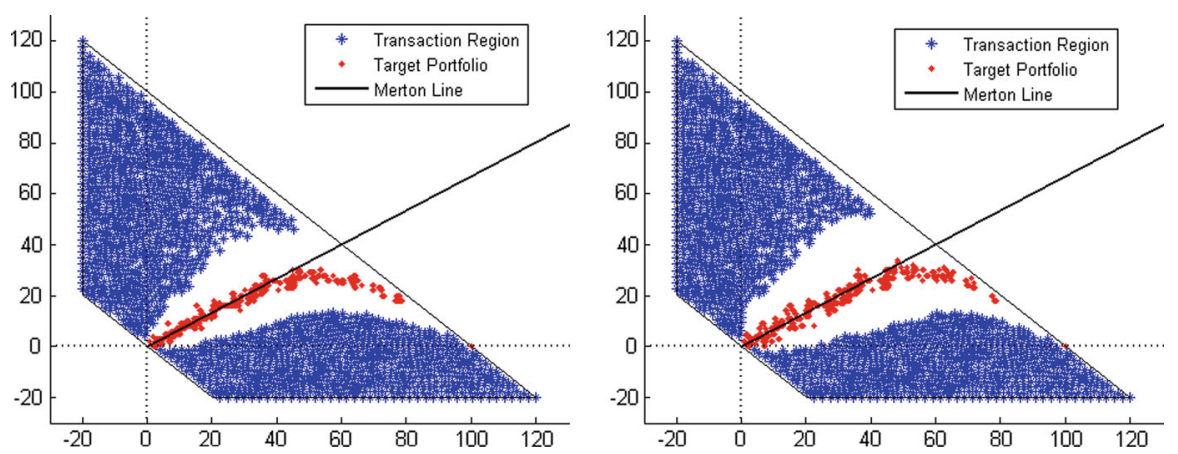

Fig. 4 Transaction area in the plane $\left(\pi_{B}, \pi_{S}\right)$ for power utility. Time $t=0$ (left) and $t=0.5$ (right). $K=0.1$

asset instead of the risky one, and the transaction area increases, since the amplitude of the no-transaction cone decreases. This implies that a more risk-averse agent recalibrates more frequently his/her portfolio, paying transaction costs, to maintain his/her portfolio into a less risky position.

To conclude, in Fig. 7 we modify the volatility parameter. Comparing this figure with Fig. 6 (down-left), where we recall that we set $\sigma=0.5$, we notice that the behavior 

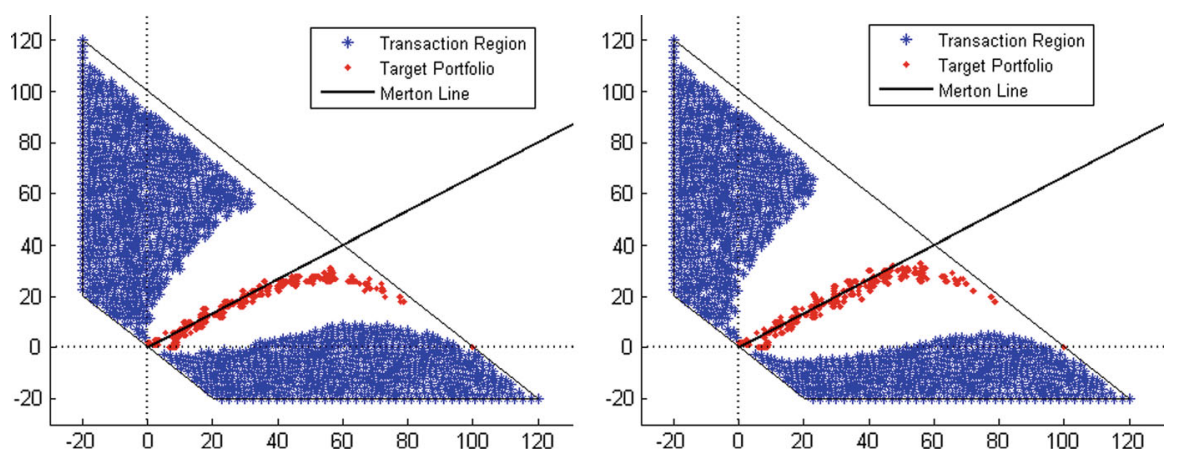

Fig. 5 Transaction area in the plane $\left(\pi_{B}, \pi_{S}\right)$ for power utility. Time $t=0$ (left) and $t=0.5$ (right). $K=0.25$
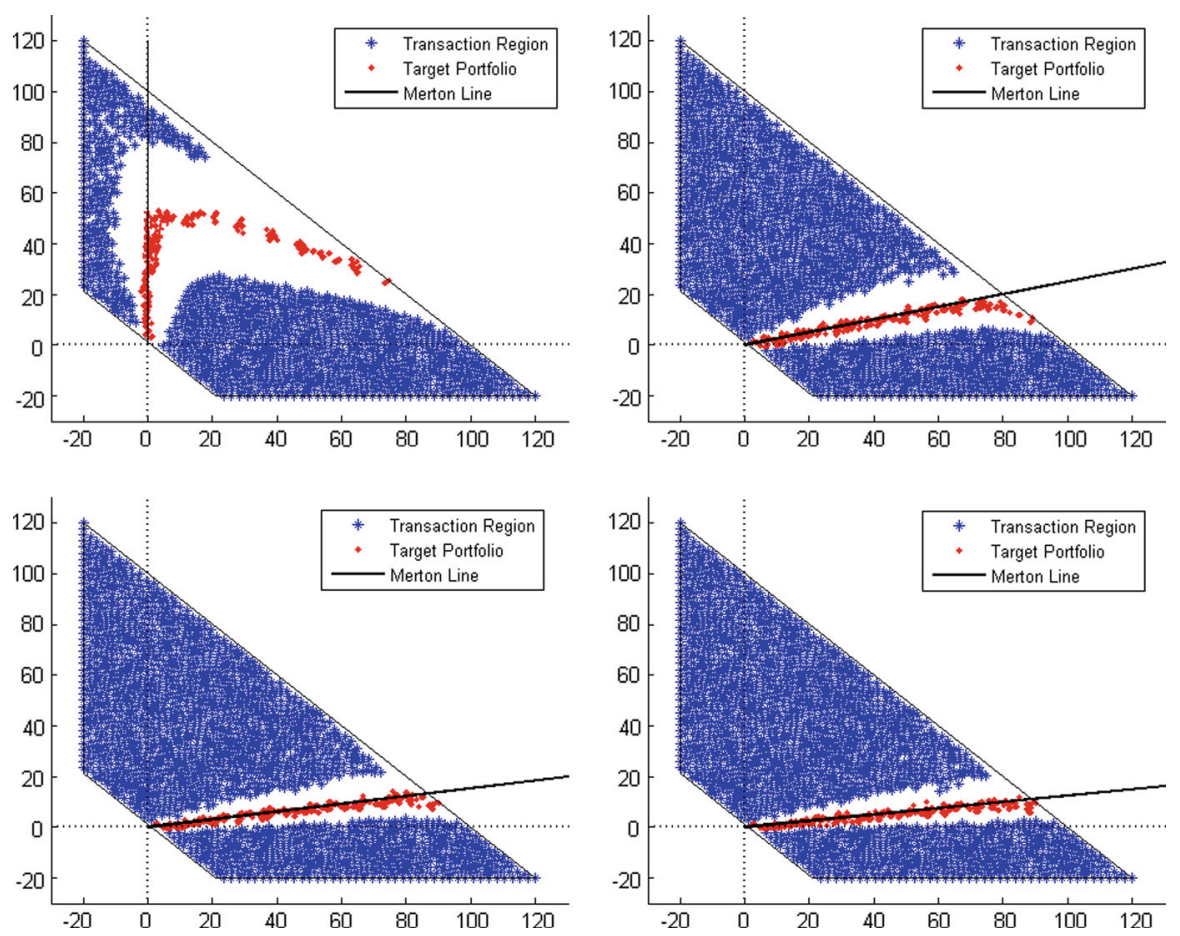

Fig. 6 Transaction area in the plane $\left(\pi_{B}, \pi_{S}\right)$ at time $t=0$ and $K=0.1$. Power utility with $\gamma=0.8$ (up-left), $\gamma=-0.5$ (down-left) and $\gamma=-0.8$ (down-right); logarithmic utility (up-right)

of the transaction area varying the volatility parameter is similar to the one described above for the risk-aversion: increasing the volatility, it is well known that the slope of the Merton's line decreases, and thus also the target portfolio line's slope decreases, while the transaction region increases. Numerical experiments not reported here show a similar behavior decreasing the risky-asset drift $b$ or increasing the risk-free interest rate $r$ : the target portfolio always follows the Merton's line and the no-trade region decreases. 

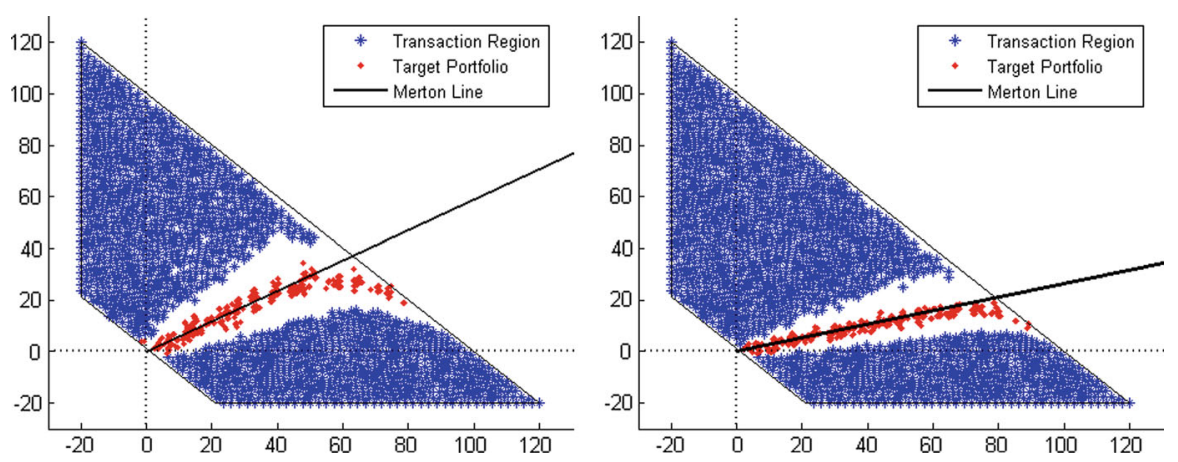

Fig. 7 Transaction area in the plane $\left(\pi_{B}, \pi_{S}\right)$ at time $t=0$ and $K=0.1$ for power utility with $\gamma=-0.5$. Volatility $\sigma=0.3($ left $)$ and $\sigma=0.4($ right $)$
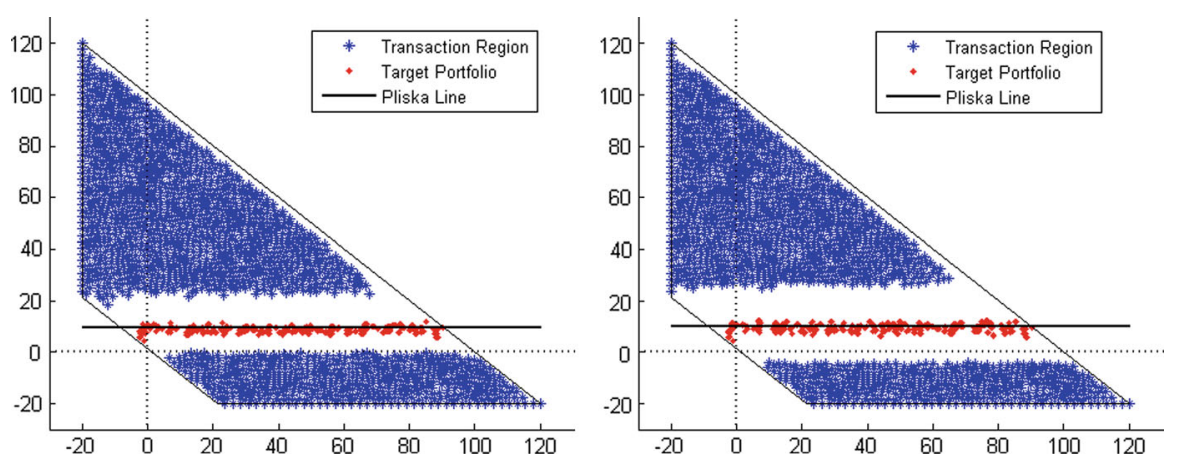

Fig. 8 Transaction area in the plane $\left(\pi_{B}, \pi_{S}\right)$ for the exponential utility. Time $t=0$ (left) and $t=0.5$ $($ right). $K=0.25$

\subsection{Exponential utility}

In this sub-section we deal with the exponential utility $u(x)=-e^{-\gamma x}$. In this case (see Pliska 1986) the optimal strategy without transaction costs is to transact continuously to maintain the amount of money invested in the risky security equal to the discounted constant

$$
\pi_{S}(t)=\frac{b-r}{\sigma^{2} \gamma} e^{-r(T-t)} .
$$

Note that this trading policy appears to be rather unrealistic. The value invested in the stock does not depend on the current wealth but only on time and the model's parameters, including the constant absolute risk-aversion $\gamma$. The investor essentially changes only the amount invested in the bank account, behaving in a more risky way when is poor than when is rich. In Figs. 8 and 9 we show the evolution of the transaction area for two different transaction costs, $K=0.25$ and $K=0.1$. The model parameters are the same as in the previous section, with the exception of $\gamma$, which is set equal to 0.02. Again, the target portfolios coincide with the optimal line without transaction costs, but the no-trade area is no more a cone, but a rectangular region with inside the 

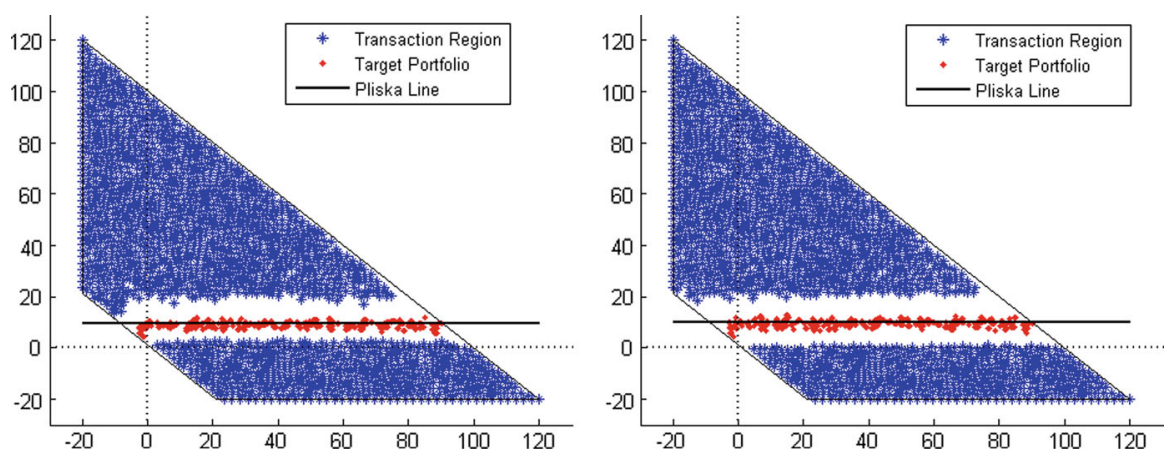

Fig. 9 Transaction area in the plane $\left(\pi_{B}, \pi_{S}\right)$ for the exponential utility. Time $t=0$ (left) and $t=0.5$ (right). $K=0.1$
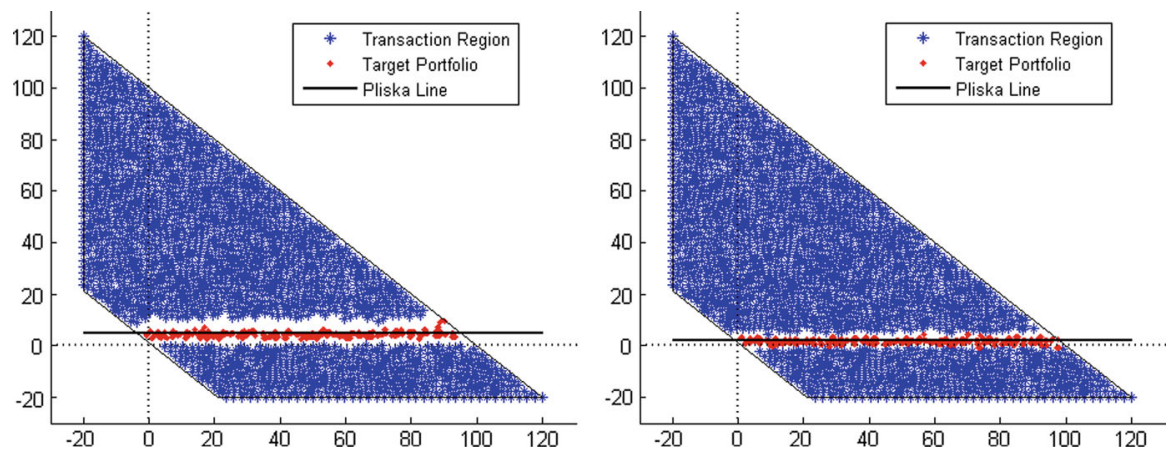

Fig. 10 Transaction area in the plane $\left(\pi_{B}, \pi_{S}\right)$ at time $t=0$ and $K=0.1$ for the exponential utility. Risk-aversion equal to $\gamma=0.04$ (left) and $\gamma=0.1$ (right)

horizontal line of optimal portfolios. Moreover, as expected, the no-transaction region is enlarging as the fixed cost and/or the time increase.

To conclude, in Fig. 10 we consider two different values of the risk-aversion parame-ter $\gamma$ : the target portfolio follows the Merton's line, moving towards the line $S=0$ as $\gamma$ increases, while the no-transaction region collapses. Numerical results not reported here show a similar behavior increasing the volatility $\sigma$, the interest rate $r$ or decreasing the drift of the risky asset $b$.

\section{Conclusions}

In this article we have studied an optimal investment problem with a fixed transaction cost and a finite horizon. In the case of a power (or logarithmic) utility we have shown that the no-transaction region closely resembles a cone, which is the same shape as the no transaction region of similar problems with proportional transaction costs (see, for instance, Davis and Norman 1990; Liu and Loewenstein 2002). The fixed cost however leads to a striking difference in the optimal policy: in our framework the optimal strategy is to move from the borders of the no-trade region to the the Merton's line, while in the proportional case the optimal strategy is to perform many infinitesimal 
transactions to remain on the boundary of the cone. It is a remarkable fact that also with the exponential utility our optimal target portfolios essentially coincide with the optimal line without transaction costs. In this case it is a horizontal line, slowly moving upwards, inside a no-trade region of a rectangular shape. Unlike most of the literature on portfolio optimization our optimal strategies are not stationary: we have shown how the no-trade regions increase as time goes on up to the finite horizon.

There are several directions in which our portfolio optimization problem under transaction costs can be further investigated. To prove rigorously that the value function is a solution of the HJBQVI it is necessary to use some kind of weak solution of this inequality, such as the very general notion of constrained viscosity solution (as for instance in Akian et al. 2001; Oksendal and Sulem 2002; Ly Vath et al. 2007). The theoretic connection between the value function and the sequence of variational inequalities also deserves additional analysis. It is likely that in the more general case of fixed plus proportional transaction costs the optimal target portfolios will split in two different lines, with possibly independent dynamics, and the coincidence with the optimal line without transaction costs will no longer hold true. All these different aspects are currently under investigation by the authors.

Acknowledgments We would like to thank two anonymous referees for useful comments, giving us the opportunity to improve the article.

\section{Appendix: Interpretation of the increasing sequence of variational inequalities}

We denote by $A^{n}\left(t, \pi_{S}, \pi_{B}\right)$ the set of admissible policies when at most $n$ interventions are admitted before the final time $T$. A policy $p=\left(\tau_{i}, \xi_{i}\right) \in A^{n}\left(t, \pi_{S}, \pi_{B}\right)$ if $p \in$ $A\left(t, \pi_{S}, \pi_{B}\right)$ and $\tau_{n+1}=+\infty$ almost surely. We introduce the value function $V^{n}$ when the set of admissible policies is restricted to $A^{n}\left(t, \pi_{S}, \pi_{B}\right)$ :

$$
V^{n}\left(t, \pi_{S}, \pi_{B}\right):=\sup _{p \in A^{n}\left(t, \pi_{S}, \pi_{B}\right)} E_{t, \pi_{S}, \pi_{B}}\left[V\left(\pi_{S}^{p}(T), \pi_{B}^{p}(T)\right)\right]=\sup _{p \in A^{n}\left(t, \pi_{S}, \pi_{B}\right)} J(p)
$$

where $\left(\pi_{S}^{p}(t), \pi_{B}^{p}(t)\right)$ is the process controlled by policy $p$, and $J(p)$ the corresponding objective value.

We define $V_{0}$ as the solution of the partial differential equation (with the other appropriate boundary conditions):

$$
\left\{\begin{array}{l}
\mathcal{L} V_{0}\left(t, \pi_{S}, \pi_{B}\right)=0 \\
V_{0}\left(T, \pi_{S}, \pi_{B}\right)=u\left(L\left(\pi_{S}, \pi_{B}\right)\right)
\end{array} .\right.
$$

Consider now the sequence of variational inequalities $(j=1, \ldots, n)$ :

$$
\left\{\begin{array}{l}
\mathcal{L} V_{j} \leq 0 \\
V_{j} \geq \mathcal{M} V_{j-1} \\
\left(V_{j}-\mathcal{M} V_{j-1}\right) \mathcal{L} V_{j}=0 \\
V_{j}\left(T, \pi_{S}, \pi_{B}\right)=u\left(L\left(\pi_{S}, \pi_{B}\right)\right)
\end{array}\right.
$$


starting from $j=1$. We show in a heuristic way that $V_{n}=V^{n}$, that is the solution of the $n-t h$ variational inequality is the value function when at most $n$ interventions are admitted.

Let $p \in A^{n}\left(t, \pi_{S}, \pi_{B}\right)$ and define $\hat{\tau}_{i}^{p}=\tau_{i}^{p} \wedge T$. By Ito's formula applied to $V_{n}$ on the interval $\left(t, \hat{\tau}_{1}\right)$, taking expectations and recalling that $\mathcal{L} V_{n} \leq 0$ (and assuming that the expectation of the stochastic integral vanishes), we obtain

$$
V_{n}\left(t, \pi_{S}, \pi_{B}\right) \geq E_{t, \pi_{S}, \pi_{B}}\left[V_{n}\left(\hat{\tau}_{1}, \pi_{S}\left(\hat{\tau}_{1}^{-}\right), \pi_{B}\left(\hat{\tau}_{1}^{-}\right)\right)\right]
$$

Moreover since $V_{n} \geq \mathcal{M} V_{n-1}$ we also have

$$
\begin{aligned}
V_{n}\left(t, \pi_{S}, \pi_{B}\right) \geq & E_{t, \pi_{S}, \pi_{B}}\left[V_{n}\left(\hat{\tau}_{1}, \pi_{S}\left(\hat{\tau}_{1}^{-}\right), \pi_{B}\left(\hat{\tau}_{1}^{-}\right)\right)\right]_{\chi_{T<\tau_{1}^{p}}} \\
& +E_{t, \pi_{S}, \pi_{B}}\left[V_{n}\left(\hat{\tau}_{1}, \pi_{S}\left(\hat{\tau}_{1}^{-}\right), \pi_{B}\left(\hat{\tau}_{1}^{-}\right)\right)\right]_{\chi_{T \geq \tau_{1}^{p}}} \\
\geq & E_{t, \pi_{S}, \pi_{B}}\left[V_{n}\left(\hat{\tau}_{1}, \pi_{S}\left(\hat{\tau}_{1}^{-}\right), \pi_{B}\left(\hat{\tau}_{1}^{-}\right)\right)\right]_{\chi_{T<\tau_{1}^{p}}} \\
& +E_{t, \pi_{S}, \pi_{B}}\left[V_{n-1}\left(\hat{\tau}_{1}, \pi_{S}\left(\hat{\tau}_{1}^{-}\right)+\xi_{1}, \pi_{B}\left(\hat{\tau}_{1}^{-}\right)-\xi_{1}-K\right)\right]_{\chi_{T \geq \tau_{1}^{p}}} \\
= & E_{t, \pi_{S}, \pi_{B}}\left[V_{n-1}\left(\hat{\tau}_{1}, \pi_{S}\left(\hat{\tau}_{1}\right), \pi_{B}\left(\hat{\tau}_{1}\right)\right)\right] .
\end{aligned}
$$

Repeating the same reasoning we obtain $(j=1, \ldots, n-1)$

$$
E_{t, \pi_{S}, \pi_{B}}\left[V_{n-j}\left(\hat{\tau}_{j}, \pi_{S}\left(\hat{\tau}_{j}\right), \pi_{B}\left(\hat{\tau}_{j}\right)\right)\right] \geq E_{t, \pi_{S}, \pi_{B}}\left[V_{n-j-1}\left(\hat{\tau}_{j-1}, \pi_{S}\left(\hat{\tau}_{j-1}\right), \pi_{B}\left(\hat{\tau}_{j-1}\right)\right)\right] .
$$

Summing up these inequalities we end with

$$
V_{n}\left(t, \pi_{S}, \pi_{B}\right) \geq E_{t, \pi_{S}, \pi_{B}}\left[V_{0}\left(\hat{\tau}_{n}, \pi_{S}\left(\hat{\tau}_{n}\right), \pi_{B}\left(\hat{\tau}_{n}\right)\right)\right] .
$$

Furthermore we have

$$
\begin{aligned}
E_{t, \pi_{S}, \pi_{B}}\left[V_{0}\left(\hat{\tau}_{n}, \pi_{S}\left(\hat{\tau}_{n}\right), \pi_{B}\left(\hat{\tau}_{n}\right)\right)\right] & \geq E_{t, \pi_{S}, \pi_{B}}\left[V_{0}\left(T, \pi_{S}^{p}(T), \pi_{B}^{p}(T)\right)\right] \\
& =E_{t, \pi_{S}, \pi_{B}}\left[u\left(L\left(\pi_{S}^{p}(T), \pi_{B}^{p}(T)\right)\right)\right]=J(p) .
\end{aligned}
$$

Therefore we have shown that

$$
V_{n}\left(t, \pi_{S}, \pi_{B}\right) \geq J(p), \quad \forall p \in A^{n}\left(t, \pi_{S}, \pi_{B}\right) .
$$

Now we show that there exists $p^{*} \in A^{n}\left(t, \pi_{S}, \pi_{B}\right)$ such that $V_{n}\left(t, \pi_{S}, \pi_{B}\right)=$ $J\left(p^{*}\right)$ and $V_{n} \equiv V^{n}$, the value function with at most $n$ interventions.

By $A^{i}, i=1, \ldots, n$, we define the set

$$
A^{i}:=\left\{\left(t, \pi_{S}, \pi_{B}\right): V_{n+1-i}=\mathcal{M} V^{n-i}\right\} .
$$


We consider the policy $p^{*}$ given recursively by $\left(\tau_{0}^{*} \equiv t, i=1, \ldots, n\right)$

$$
p^{*}=\left\{\begin{array}{l}
\tau_{i}^{*}=\left\{\begin{array}{l}
\inf \left\{I_{i} \equiv\left\{T \geq s \geq \tau_{i-1}^{*}:\left(s, \pi_{S}\left(s^{-}\right), \pi_{B}\left(s^{-}\right)\right) \in A^{i}\right\}\right\} \text { if } I_{i} \neq \emptyset \\
+\infty \text { if } I_{i}=\emptyset
\end{array}\right. \\
\xi_{i}^{*}=\left\{\begin{array}{l}
\arg \max _{\xi \in \mathbb{R}} V_{n-i}\left(\tau_{i}^{*}, \pi_{S}\left(\tau_{i}^{*-}\right)+\xi, \pi_{B}\left(\tau_{i}^{*-}\right)-\xi-K\right) \text { if } \tau_{i}^{*}<+\infty \\
\operatorname{arbitrary} \text { if } \tau_{i}^{*}=+\infty
\end{array}\right.
\end{array}\right.
$$

It is not difficult to see that using this policy the above inequalities become equalities and we have

$$
V^{n}\left(t, \pi_{S}, \pi_{B}\right)=J\left(p^{*}\right)=V_{n}\left(t, \pi_{S}, \pi_{B}\right)
$$

\section{References}

Achdou Y, Pironneau O (2005) Computational methods for option pricing. Frontiers in Applied Mathematics, vol 30. SIAM, Philadelphia

Akian M, Menaldi JL, Sulem A (1996) On an investment-consumption model with transaction costs. SIAM J Control Optim 34(1):329-364

Akian M, Sulem A, Taksar M (2001) Dynamic optimization of long-term growth rate for a portfolio with transaction costs and logarithmic utility. Math Finance 11(2):153-188

Assaf D, Klass M, Taksar M (1988) A diffusion model for optimal portfolio selection in the presence of brokerage fees. Math Oper Res 13:277-294

Baccarin S (2009) Optimal impulse control for a multidimensional cash management system with generalized cost functions. Eur J Oper Res 196(1):198-206

Barucci E, Marazzina D (2012) Optimal investment, stochastic labor income and retirement. Appl Math Comput 218-9:5588-5604

Bielecki T, Chancelier J, Pliska S, Sulem A (2004) Risk sensitive portfolio optimization with transaction costs. J Comput Finance 8:39-63

Cadenillas A (2000) Consumption-investment problems with transaction costs: survey and open problems. Math Method Oper Res 51:43-68

Chancelier J, Oksendal B, Sulem A (2002) Combined stochastic control and optimal stopping, and application to numerical approximation of combined stochastic and impulse control. Proc Steklov Inst Math 237:140-163

Davis M, Norman A (1990) Portfolio selection with transaction costs. Math Oper Res 15:676-713

Dumas B, Luciano E (1991) An exact solution to a dynamic portfolio choice problem. J Finance 46:577-595

Eastham JE, Hastings KJ (1988) Optimal impulse control of portfolios. Math Oper Res 13:588-605

Korn R (1998) Portfolio optimization with strictly positive transaction costs and impulse control. Financ Stoch 2:85-114

Ly Vath V, Milf M, Pham H (2007) A model of optimal portfolio selection under liquidity risk and price impact. Finance Stoch 11:51-90

Liu H, Loewenstein M (2002) Optimal portfolio selection with transaction costs and finite horizon. Rev Financ Stud 15-3:805-835

Marazzina D, Reichmann O, Schwab Ch (2012) hp-DGFEM for Kolmogorov-Fokker-Planck equations of multivariate Lévy processes. MAS 22-1:115005.1-37

Merton RC (1969) Lifetime portfolio selection under uncertainty: the continuous time case. Rev Econ Stat 51:247-257

Morton AJ, Pliska SR (1995) Optimal portfolio management with transaction costs. Math Finance 5:337356

Oksendal B, Sulem A (2002) Optimal consumption and portfolio with both fixed and proportional Costs. SIAM J Control Optim 40(6):1765-1790

Pliska SR (1986) A stochastic calculus model of continuous trading: optimal portfolios. Math Oper Res $11: 371-382$ 
Shreve S, Soner H (1994) Optimal investment and consumption with transaction costs. Ann Appl Probab 4:609-692

Wilmott P, Howison S, Dewynne J (1995) The mathematics of financial derivatives: a student introduction. Cambridge University Press, Cambridge 